\title{
On the Road to Resolution: Do People know where to go to resolve their Untreatable Chronic Headache?
}

\author{
Barreiros HP*1, Souza $\mathrm{J}^{2}$, and Moreira $\mathrm{P}^{3}$ \\ ${ }^{1}$ Master Program, Headache Section, Fluminense Federal University (UFF), Brazil. \\ ${ }^{2}$ Associate Professor, Neurology Department, Fluminense Federal University (UFF), Brazil. \\ ${ }^{3}$ Adjunct Professor, Antonio Pedro University Hospital, (HUAP), Fluminense Federal University (UFF), Brazil.
}

Received: February 19, 2018; Published: February 27, 2018

*Corresponding author: Barreiros HP, Rua Francisco Sá 336-402, Varzea, Teresópolis, RJ, Brazil, Tel: 55-21-9-8669-9874/ 55-21-2742-2996;

Email: hpbarreiros@uol.com.br

\section{Abstract}

Although headache is a common disorder with a great burden on quality of live, there are many who have not been able to solve it yet. Partially due to the lack of understanding about who is the physician more trained regarding this disturbance. The following text reports a series of people in quest to resolve their untreatable chronic headache.

Keywords: Headache, Untreatable, Education

Opinion

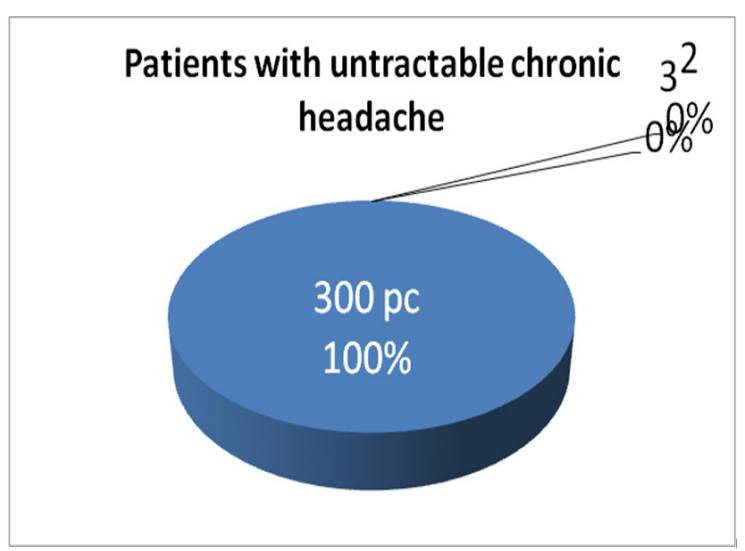

Figure 1: They had never been in a neurologist consult before but they had already been in consult with nonneurologist physicians and even with psychologists previously.

Three hundred patients, aged 8-74 years-old, have looked for a neurologist appointment because of an untreatable chronic headache. They had never been in a neurologist consult before but they had already been in consult with non-neurologist physicians and even with psychologists previously (Figure 1). Obviously none of them had managed to get free of their pain so that they had come to the specialist as a hope for healing. Neurologists are considered to be the health professionals more acquainted to the complaint of headache. Regardless the diagnosis of the headaches, which prevalence are well known [1], the purpose of these work was to identify the current opinion that has been prevalent among the suffering patients regarding which doctor should they attend. Behind this experience lies the hidden idea of what do ordinary people think that their headaches are for.

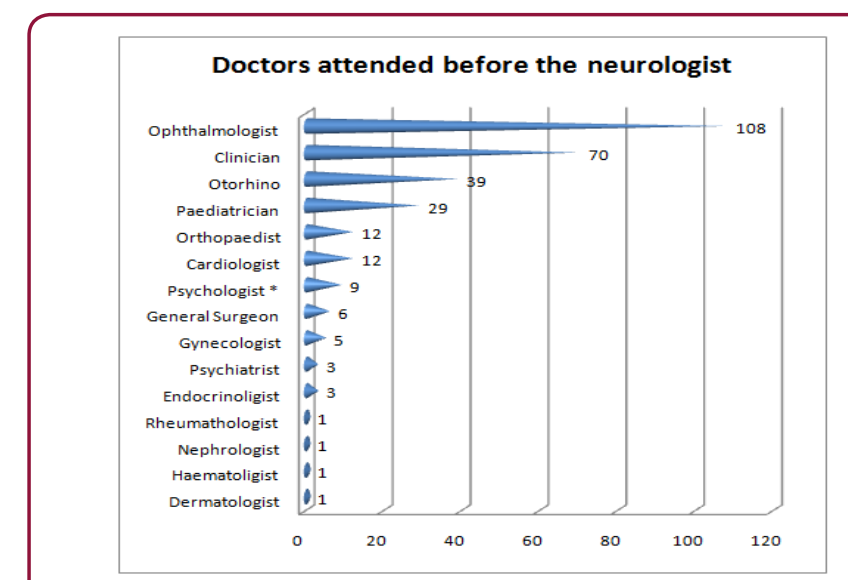

Figure 2: Three persons went to a psychiatrist or to an endocrinologist, and finally one person were in search either for a rheumatologist or a nephrologist, a haematologist or even a dermatologist. 
Out of this total of 300 patients, 108 (one hundred and eight) had been to an ophthalmologist before, as their first choice for medical help. In the next sample, 70 (seventy) patients had looked for a clinician as the starting consult. Then, 39 patients were in search for an otorhino, first, and 29 went to see a paediatrician before. Twelve people had started with a cardiologist and an orthopaedist. Six persons went to see a general surgeon and five, a gynecologist. Three persons went to a psychiatrist or to an endocrinologist, and finally one person was in search either for a rheumatologist or a nephrologist, a haematologist or even a dermatologist (Figure 2).

Itis note worthy thatnine people (3\%) have had their initial quest with a psychologist, who is a non-physician health professional. In an average percentage, $36 \%$ had looked for an ophthalmologist, $23 \%$ for a clinician and $13 \%$ for an otorhino, at the beginning. Ten per cent of patients had started with a paediatrician and four per cent with either a cardiologist or an orthopaedist. Three per cent went to a psychologist, two per cent to a general surgeon, and one per cent to a psychiatrist or an endocrinologist. Less than one per cent ( 1 person only) had started with a haemathologist or a rheumatologist or nephrologist or even a dermatologist (Figure 3). This values have shown that there was a wide spread idea among lay people that headaches are caused mainly by diseases under the scope of non-neurologists doctors, like refractive errors of vision, rhino sinusitis, anxiety or depression, high blood pressure or even cerebral tumors. Although these quoted situations may be common co-morbidities [2], they usually are not the cause of most of the untreatable chronic headaches and, thus, remain without the correct approach and without the adequate solution.

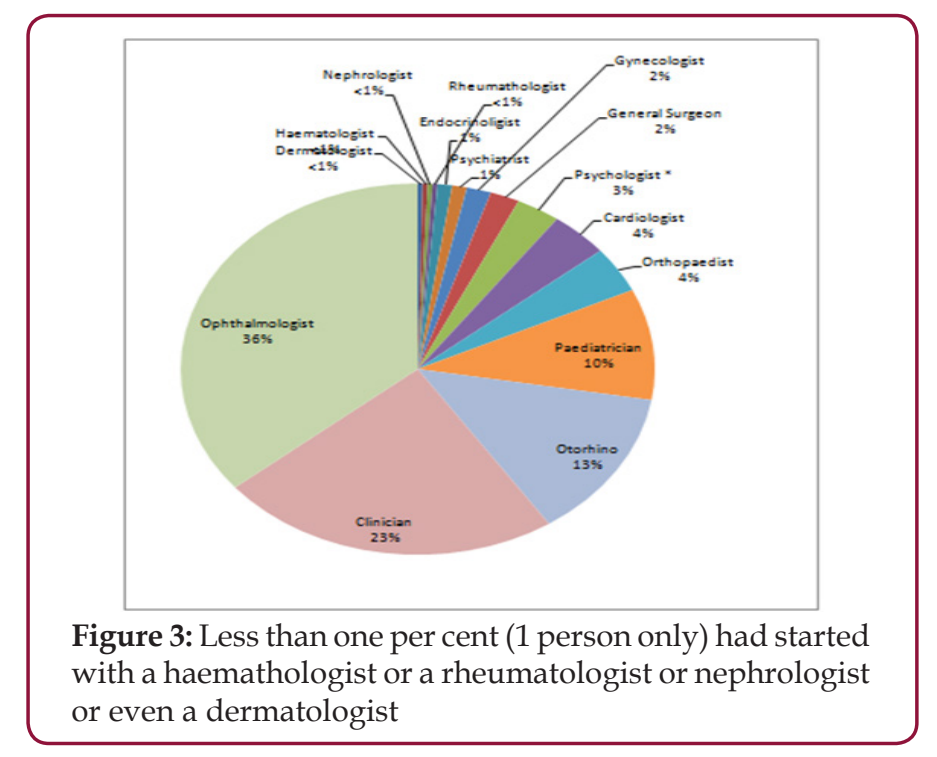

It is noteworthy again that most of these headaches are either treatable and even preventable if adequately managed. Are ordinary people in need of more knowledge? Should the government or the media provide more adequate information about these potentially treatable and preventable source of pain and distress? Surely yes! [3]

\section{References}

1. Information adapted from the World Health Organization.

2. Rigmor Jensen,Lars J Stovner, (2008) Epidemiology and comorbidity of headache $7(4): 354-361$.

3. International Head Ache Society.

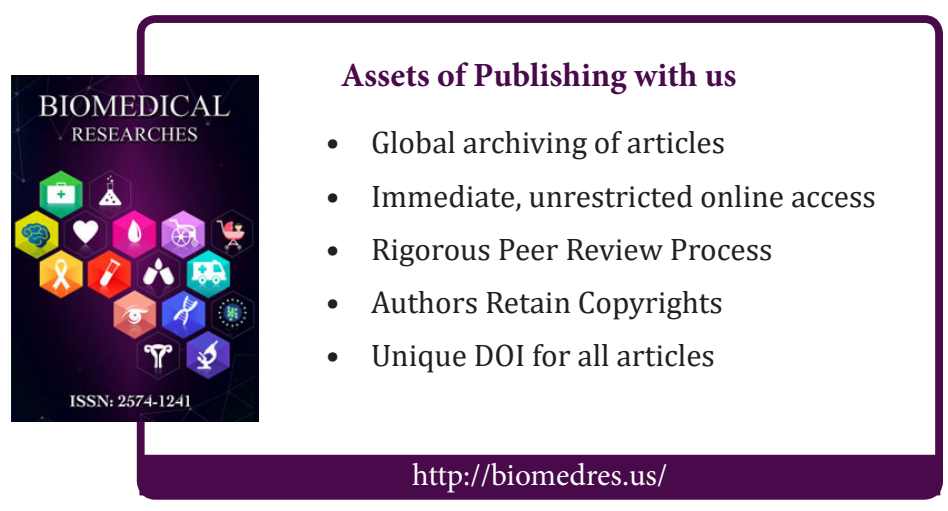

